\title{
Turbo Equalization With Irregular Turbo Codes
}

\author{
Vladimir D. Trajković ${ }^{1}$, Minyue Fu ${ }^{2}$, Peter J. Schreier ${ }^{3}$ \\ School of Electrical Engineering and Computer Science, \\ The University of Newcastle \\ Callaghan 2308, NSW, AUSTRALIA \\ ${ }^{1}$ Vladimir.Trajkovic@newcastle.edu.au \\ ${ }^{2}$ Minyue.Fu@newcastle.edu. au \\ ${ }^{3}$ Peter.Schreier@newcastle.edu.au
}

\begin{abstract}
We analyze a turbo equalization system that combines Maximum a Posteriori Probability (MAP) equalization with irregular turbo codes. Our goal is to approach the information capacity limit for severe Inter-Symbol Interference (ISI) channels. To this end, we optimize the degree profile of irregular turbo codes by maximizing the minimum distance between the mutual information transfer functions for the MAP equalizer and decoder. We show that turbo equalizers employing such optimized irregular turbo codes can approach the information capacity limit of some severe ISI channels within $0.75 \mathrm{~dB}$.
\end{abstract}

\section{INTRODUCTION}

Inter-Symbol Interference (ISI) is one of the major impediments for attaining high-speed data transmission in modern communication systems. Channel equalization [1] and channel coding [2] are two techniques that have been used for decades for reliable data transmission. However, in a standard approach, these two techniques are usually applied consecutively. Turbo equalization, on the other hand, is a detection method that combines channel equalization and channel decoding in an iterative way, so that equalizer and decoder successively exchange information. Turbo equalization was originally proposed in [3], and named after its similarity with turbo codes [4]. The original turbo equalizer [3], [5] combines Maximum Likelihood (ML) equalization and ML decoding in a serially concatenated scheme similar to Serially Concatenated Convolutional Codes [6].

Alternative low-complexity turbo equalization implementations have been studied in [7]-[11]. However, the reduction in complexity is obtained at the expense of SNR-BER performance, which is usually about $2 \mathrm{~dB}$ at a BER of $10^{-5}$ [9].

A turbo equalization scheme that combines ML equalization and turbo decoding was proposed in [12], and further investigated in [13]. The convergence properties of three-stage turbo equalization were analyzed in [14], and capacity approaching performance was reported. However, this performance was estimated using three-dimensional (3D) Extrinsic Information Transfer (EXIT) chart analysis [15], without running Monte Carlo simulations.

In this paper, we aim to design turbo equalizers that approach the information capacity limit of severe ISI channels.

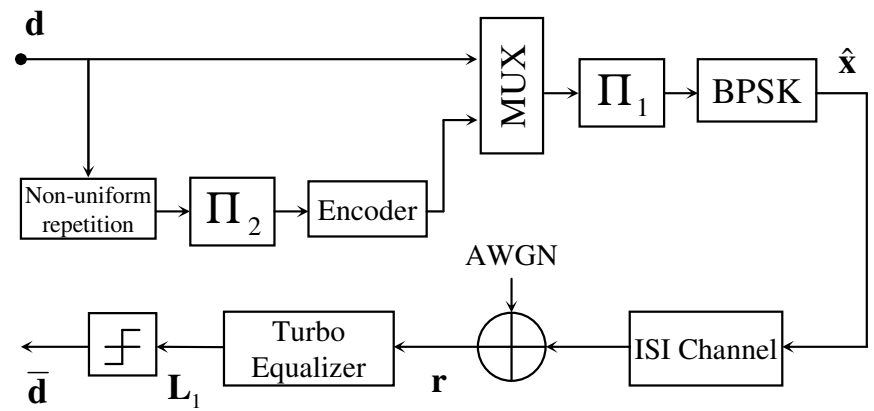

Fig. 1. System model

For this purpose, we analyze turbo equalizers that employ MAP equalization and irregular turbo codes. Using EXIT chart analysis, we optimize the degree profile of irregular turbo codes [16]. We show that, for certain severe ISI channels, it is possible to approach the information capacity limit within $0.75 \mathrm{~dB}$. This gives an improvement of about $0.1 \mathrm{~dB}$ compared to regular turbo codes.

While previous work on turbo equalization [7]-[11] has compared the BER performance with the performance of the coded system on an AWGN channel, our performance comparisons are made in terms of information capacity. The comparisons in [7]-[11] are dependent on the employed channel codes and do not show how close the performance is to the channel capacity. In order to calculate the information capacity limits for ISI channels in terms of BER-SNR, we use the algorithm presented in [17] combined with the approach in [16, p.129].

The remainder of the paper is organized as follows. In Section II, we describe the system model, and in Section III, we explain how to optimize irregular turbo codes. Section IV presents simulation results, and the paper is concluded in Section V.

\section{System MOdeL}

The system model is shown in Fig. 1. On the transmitter side, the information bits are encoded with an irregular turbo code, as proposed in [16]. The encoded sequence consists of $K$ 


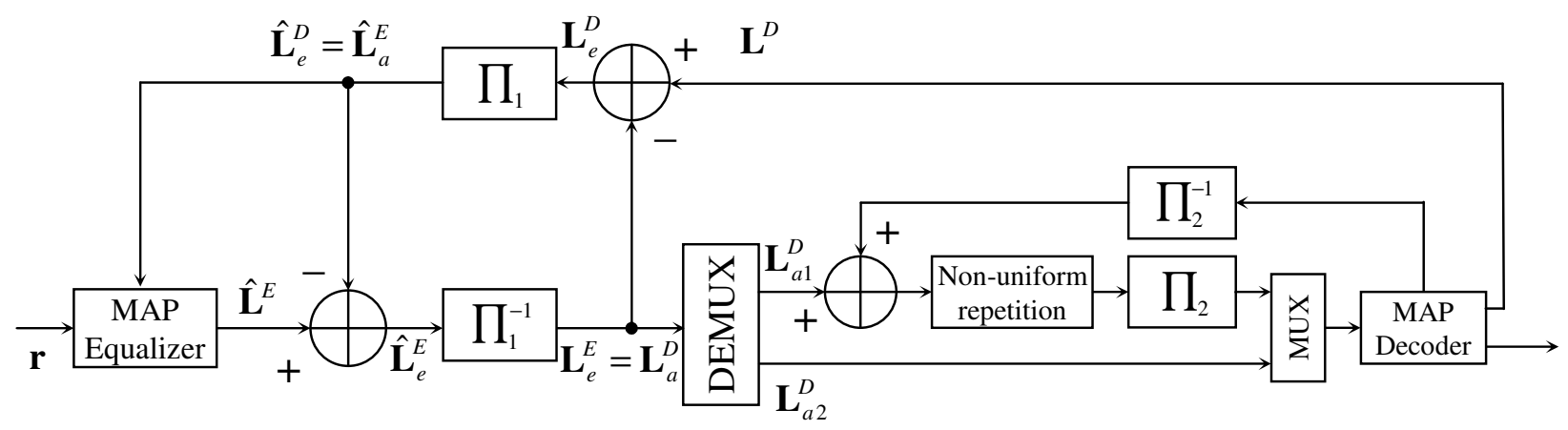

Fig. 2. Turbo equalizer employing turbo decoding

systematic information bits, and additional parity check bits. Irregular turbo codes are obtained from regular turbo codes by selecting a degree profile $f_{d} \in[0,1], d \in\{2, \ldots, D\}$, which satisfies $\sum_{d=2}^{D} f_{d}=1$. In this degree profile, $f_{d}$ specifies the fraction of systematic bits that is repeated $d$ times. The sequence of repeated systematic bits is then fed into an interleaver denoted by $\Pi_{2}$. The interleaved sequence is encoded by a recursive systematic convolutional (RSC) code of rate $r$. For a regular turbo code, all $K$ systematic bits are copied exactly twice. After RSC encoding, this produces $K(1 / r-1)$ parity check bits, giving an overall code rate of $R=1 /(1+2(1 / r-1))$. In order to obtain a desired turbo code rate less than $R$, puncturing of parity check bits is performed. On the other hand, for an irregular turbo code, the total number of parity check bits is $K^{\prime}(1 / r-1)$, with

$$
K^{\prime}=K \cdot \sum_{d=2}^{D} f_{d} \cdot d,
$$

which gives a new overall code rate

$$
R^{\prime}=\frac{K}{K+\left(\frac{1}{r}-1\right) K^{\prime}}=\frac{1}{1+\left(\frac{1}{r}-1\right) \sum_{d=2}^{D} f_{d} \cdot d}
$$

In the general case $D>2$, we have $R^{\prime}<R$, which means additional puncturing has to be introduced in order to keep the desired turbo code rate. The systematic bits and parity check bits together make up a sequence of coded bits. The coded bits are interleaved using another interleaver, denoted by $\Pi_{1}$. In this paper, we refer to the interleaver $\Pi_{1}$ as the outer interleaver and " $"$ will be used to denote a sequence interleaved by $\Pi_{1}$. Interleaver $\Pi_{2}$ is referred to as the inner interleaver. Interleaved coded bits are BPSK modulated and the symbols are transmitted through an ISI channel. The received signal at time instant $k$ is given by

$$
y_{k}=\sum_{i=0}^{M-1} h_{i} \cdot \hat{x}_{k-i}+w_{k},
$$

where $M$ is the length of the channel impulse response, $h_{0}, h_{1}, \ldots, h_{M-1}$ are discrete channel coefficients, $\hat{x}_{k}$ is the $k$ th transmitted coded bit after outer interleaving, and $w_{k}$ is a sample of Additive White Gaussian Noise (AWGN). The received signal is detected by a turbo equalizer. The frontend of the turbo equalizer is a Maximum a Posteriori Probability (MAP) equalizer. At iteration $n$, it delivers Log Likelihood Ratios (LLRs) for each transmitted bit as

$$
\hat{L}^{E(n)}\left(\hat{x}_{k}\right)=\log \frac{p\left(\hat{x}_{k}=1 \mid \mathbf{r}, \hat{\mathbf{L}}_{a}^{E(n)}\right)}{p\left(\hat{x}_{k}=-1 \mid \mathbf{r}, \hat{\mathbf{L}}_{a}^{E(n)}\right)} .
$$

In this equation, $\hat{\mathbf{L}}_{a}^{E(n)}$ is the equalizer's a priori information at iteration $n$, which is the extrinsic information delivered by the turbo decoder at the previous iteration $n-1$, i.e., $\hat{\mathbf{L}}_{a}^{E(n)}=$ $\hat{\mathbf{L}}_{e}^{D(n-1)}$. It is calculated as

$$
\hat{L}_{a}^{E(n)}\left(\hat{x}_{k}\right)=\hat{L}^{D(n-1)}\left(\hat{x}_{k}\right)-\hat{L}_{e}^{E(n-1)}\left(\hat{x}_{k}\right) .
$$

At the first turbo equalization iteration, $\hat{L}_{a}^{E(n)}=0$. After a certain number of turbo decoding (inner) iterations, the turbo decoder delivers LLRs for both systematic and parity check bits using the MAP algorithm described in [18]. The decoding MAP algorithm calculates LLRs based on the extrinsic information from the equalizer, which is used as a priori information in the decoder, i.e., $\mathbf{L}_{a}^{D(n)}=\mathbf{L}_{e}^{E(n)}=\mathbf{L}^{E(n)}-\mathbf{L}_{e}^{D(n-1)}$. The stream $\mathbf{L}_{a}^{D(n)}$ is split into two sub-streams: $\mathbf{L}_{a 1}^{D(n)}$, which contains a priori information for the systematic bits, and $\mathbf{L}_{a 2}^{D(n)}$, which contains a priori information for the parity check bits. The $L$-values for the systematic bits, $\mathbf{L}_{a 1}^{D(n)}$, are repeated by the non-uniform repetition block. At the initial turbo decoding iteration, $L$-values obtained from the equalizer are repeated for all systematic bits according to the degree profile. For example, if systematic bit $i$ is repeated $d$ times, and the corresponding $L$ value delivered by the MAP equalizer is $L_{i}^{0}$, at the first iteration we set

$$
L_{i, k}=L_{i}^{0}, \quad k=1, \ldots, d
$$

This sequence is then mixed with the $L$-values $\mathbf{L}_{a 2}^{D(n)}$ and used as the a priori information in the MAP decoder. During the (inner) turbo decoding process, the MAP decoder delivers the extrinsic information for systematic bits only. For the $k$-th repetition of bit $i$, the extrinsic information at iteration $n$ is calculated as

$$
\left(L_{e}\right)_{i, k}^{n}=L_{i, k}^{n}-L_{i, k}^{0}-\left(L_{e}\right)_{i, k}^{n-1}
$$


where $L_{i, k}^{n}$ is the a posteriori $L$-value for the $k$-th repetition of bit $i$. The a priori information $\left(L_{a}\right)_{i, k}^{n+1}$ is then obtained as

$$
\left(L_{a}\right)_{i, k}^{n+1}=\left(L_{e}\right)_{i, k}^{n}=L_{i, k}^{n}-L_{i, k}^{0}-\left(L_{e}\right)_{i, k}^{n-1} .
$$

The sequence thus obtained is then interleaved using the inner interleaver, and the $L$-values for the parity check bits are added. After a certain number of inner turbo iterations, the turbo decoder delivers LLRs for all systematic and parity check bits. This stream is denoted $L^{D}$ in Fig. 2. The extrinsic information delivered by the turbo decoder is obtained by calculating $L^{D}-L_{a}^{D}$. This extrinsic information is then interleaved and used in the MAP equalizer at the following turbo equalization iteration as a priori information. After the final outer iteration, hard decisions on systematic bits only are made as $\overline{\mathbf{d}}=\operatorname{sign}\left(\mathbf{L}_{1}\right)$ in order to retrieve the transmitted information.

\section{OPTIMIZED IRREGULAR TURBO CODES}

In this section, we perform an optimization of degree profiles based on the extrinsic information transfer (EXIT) between the constituent components of the turbo equalizer. We work with the transfer functions $I_{o}^{E}=T_{E}\left(I_{i}^{E}\right)$ and $I_{o}^{D}=T_{D}\left(I_{i}^{D}\right)$. The functions $I_{i}^{D}$ and $I_{o}^{D}$ are defined as $I_{i}^{D}=I_{o}^{E}=I\left(L_{e}^{E} ; X\right)$ and $I_{o}^{D}=I_{i}^{E}=I\left(L_{e}^{D} ; X\right)$, where $I(\cdot ; \cdot)$ denotes mutual information. Mutual information between $L$-values (a priori or extrinsic information) and transmitted bits can be found as

$$
\begin{aligned}
I(L ; X) & =\frac{1}{2} \sum_{x= \pm 1} \int_{-\infty}^{+\infty} p_{L}(\xi \mid X=x) \\
& \times \log _{2} \frac{2 p_{L}(\xi \mid X=x)}{p_{L}(\xi \mid X=-1)+p_{L}(\xi \mid X=+1)} d \xi .
\end{aligned}
$$

where $p_{L}(\xi \mid X=x)$ is the probability density function of an $L$-value. We use the assumption that the input $L$-values are Gaussian. For the extrinsic information (output $L$-values), we use the observation from [19], [20] that the mutual information between the original binary sequence and an $L$-sequence can be approximated as closely as desired by the time average

$$
I(L ; X) \approx 1-\frac{1}{N} \sum_{i=1}^{N} \log _{2}\left(1+e^{-x_{i} \cdot L_{i}}\right) .
$$

We observe that both the input a priori information and the turbo decoding transfer function are dependent on the degree profile of the irregular turbo code. Our goal is to optimize the degree profile in order to provide the lowest turbo detection threshold. For this purpose, we first apply curve fitting to obtain the functions $T_{E}(x)$ and $T_{D}(x)$, where $x$ stands for the input a priori information. Then we perform the optimization

$$
\left\{f_{2}, \ldots, f_{D}\right\}=\arg \max _{f_{2}, \ldots, f_{D}} \min _{x}\left(T_{E}(x)-T_{D}^{-1}\left(f_{2}, \ldots, f_{D}, x\right)\right) .
$$

We limit our analysis to the case when there is only one nonzero degree $d>2$, in addition to degree $d=2$. However, our analysis can easily be extended to any number of degrees. We use an approach similar to that in [21], i.e., we fix the code rate $R^{\prime}$ and then find the mutual information transfer function of the MAP decoder for different values for the degree $d>2$. We consider $d \in\{6,7, \ldots, 12\}$, as in [21]. Because $R^{\prime}$ is fixed,

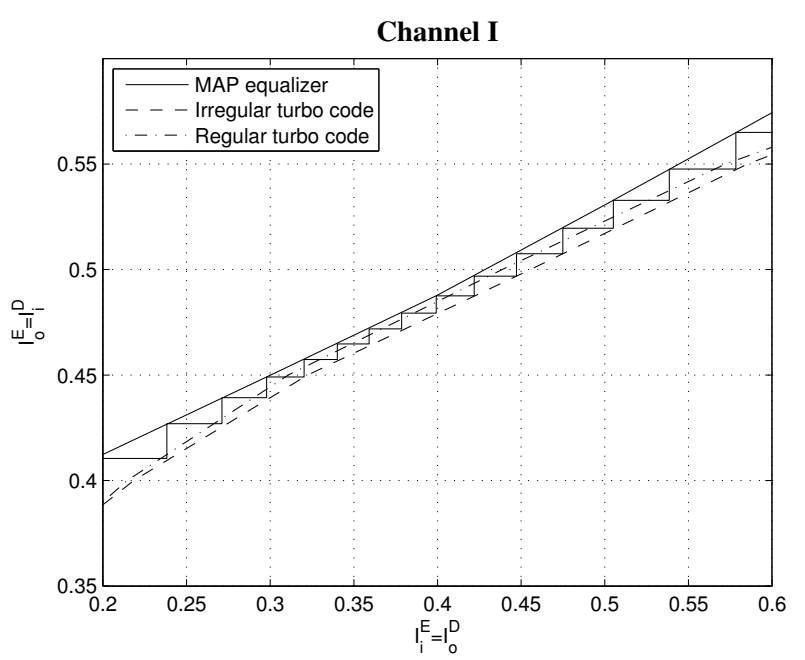

Fig. 3. Turbo equalizer EXIT chart for Channel I and $E_{b} / N_{0}=3.6 \mathrm{~dB}$. The capacity limit for channel I is $2.95 \mathrm{~dB}$.

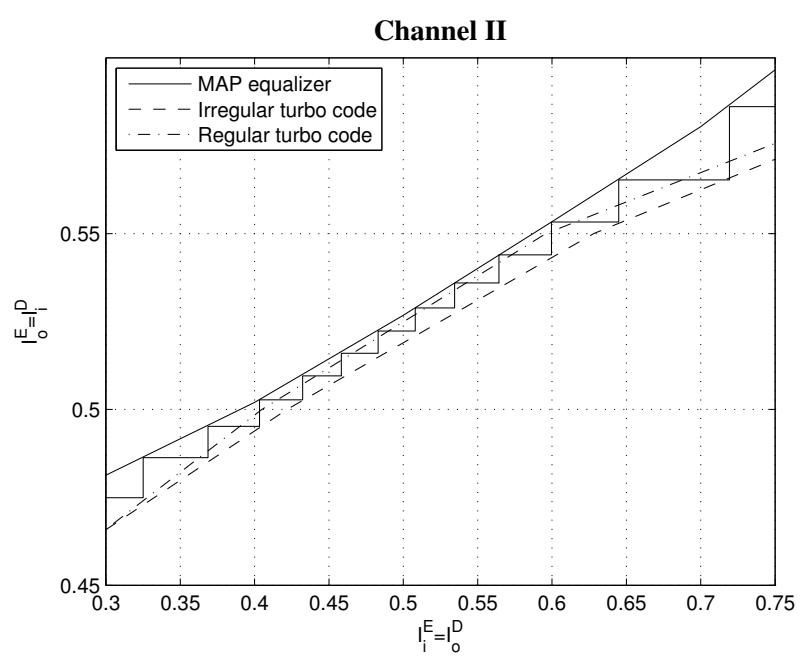

Fig. 4. Turbo equalizer EXIT chart for Channel II and $E_{b} / N_{0}=2.5 \mathrm{~dB}$. The capacity limit for channel II is $2 \mathrm{~dB}$.

the encoder always produces the same number of parity check bits, which enables the use of the same puncturing pattern independent of the degree profile. The functions $T_{E}(x)$ and $T_{D}(x)$ depend on a variety of design parameters such as RSC code parameters, number of different degrees, and puncturing patterns. The following section explains the selection of these parameters in more detail.

\section{Simulation Results}

In this section, we present simulation results obtained for two different ISI channels. The first channel, commonly used in the turbo equalization literature, is $h_{1}=$ $\left[\begin{array}{lllll}0.227 & 0.46 & 0.688 & 0.46 & 0.227\end{array}\right]^{T}$ from [1]. We refer to this channel as Channel I. The second channel is $h_{2}=$ $\left[\begin{array}{lllll}\sqrt{0.45} & \sqrt{0.25} & \sqrt{0.15} & \sqrt{0.1} & \sqrt{0.05}\end{array}\right]^{T}$ from [3]. We refer to this channel as Channel II. In both examples, an RSC code with rate $r=1 / 2$ is used to encode the systematic bits 
after non-uniform repetition. For a regular turbo code, this gives code rate $R=1 / 3$, and the overall turbo code rate $1 / 2$ is obtained by appropriate puncturing. For irregular codes, the code rate $R^{\prime}$ is fixed at $5 / 17$, which is slightly lower than $1 / 3$. The choice of this code rate $R^{\prime}$ is adopted from [16]. The overall code rate $1 / 2$ in the irregular case is also obtained by appropriate puncturing.

As mentioned before, we limit our analysis to two degrees. In all cases, one degree is $d=2$ and the second degree $d$ is chosen from the set $\{6,7, \ldots, 12\}$. Then we find the transfer functions $T_{D}(x)$ for the turbo code for the different degree profiles and choose the one corresponding to (11).

The EXIT chart describing the turbo equalization of Channel I, employing regular and irregular turbo codes of code rate $R=1 / 2$ using generator polynomials [3 2 ] at $E_{b} / N_{0}=3.6 \mathrm{~dB}$, is shown in Fig. 3. Figure 4 shows a section of an EXIT chart for Channel II at $E_{b} / N_{0}=2.5 \mathrm{~dB}$, with generator polynomials [7 6]. The generator polynomials were determined through an extensive search for good constituent codes for regular turbo codes employed within the turbo equalizer for these channels. For both channels, we find that the degree profile that optimizes the performance according to (11) is $f_{2}=0.9555$ and $f_{11}=0.0455$.

In each simulation, we generated blocks of approximately $2^{18}$ information bits, which after channel coding and modulation gives $2 \cdot 2^{18}$ BPSK symbols. Since the code is irregular, it is not generally possible to generate blocks of exactly $2^{18}$ bits. The coded sequence is interleaved using a random interleaver. All BER simulations were performed until 100 erroneous packets had been collected.

Figure 5 shows the BER performance of the turbo equalizer on channel I, employing regular and irregular turbo codes with the generator polynomials [3 2]. The BER performance is compared with the information capacity limit for Channel I, which is around $E_{b} / N_{0}=2.95$ at low BER $\left(\leq 10^{-5}\right)$. This information capacity limit was calculated using the algorithm presented in [17], combined with the approach in [16, p.129]. The irregular turbo code requires an $E_{b} / N_{0}$ of $3.7 \mathrm{~dB}$ to achieve a BER less than $10^{-5}$. This is $0.75 \mathrm{~dB}$ from the capacity limit and about $0.1 \mathrm{~dB}$ less than the regular turbo code. The number of outer iterations required to achieve this BER is $n_{\text {out }}=28$.

For the irregular code, we had to generate 426 blocks in order to collect 100 erroneous packets. For the regular code, the number of generated blocks is 405 . In order to reduce the computational complexity, we set the number of inner iterations to $n_{\text {in }}=5$ for $n_{\text {out }}$ between 1 and $7, n_{\text {in }}=10$ for $n_{\text {out }}$ between 8 and 20 , and $n_{\text {in }}=20$ for $n_{\text {out }}>20$. This setup is based on our finding that an increase in the number of inner iterations beyond five provides negligible improvement when the turbo equalizer operates below the bottleneck region $\left(I_{i}^{D}<0.4\right)$.

Figure 6 shows a similar analysis for Channel II. The capacity limit for this channel and code rate $1 / 2$ is around 2 $\mathrm{dB}$. For this channel, the irregular turbo-coded turbo equalizer requires $E_{b} / N_{0}=2.55 \mathrm{~dB}$ to achieve a BER of less than

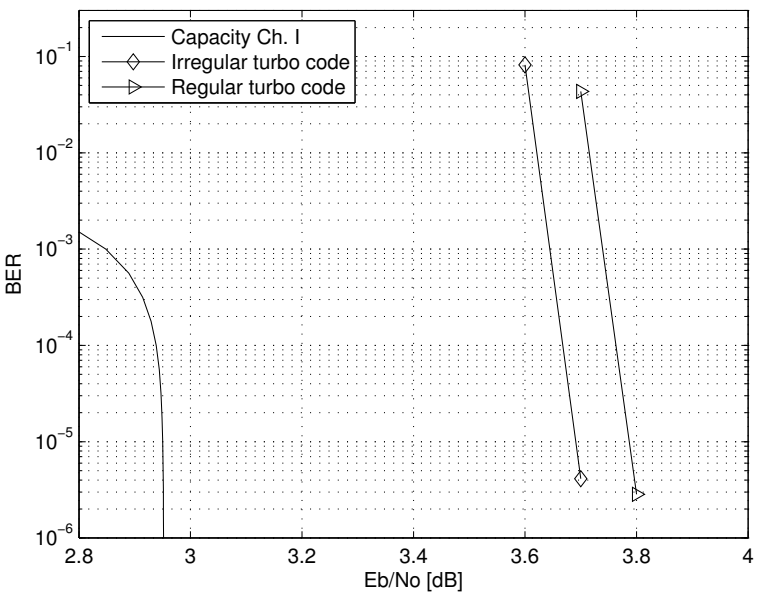

Fig. 5. Turbo equalization BER results for Channel I.

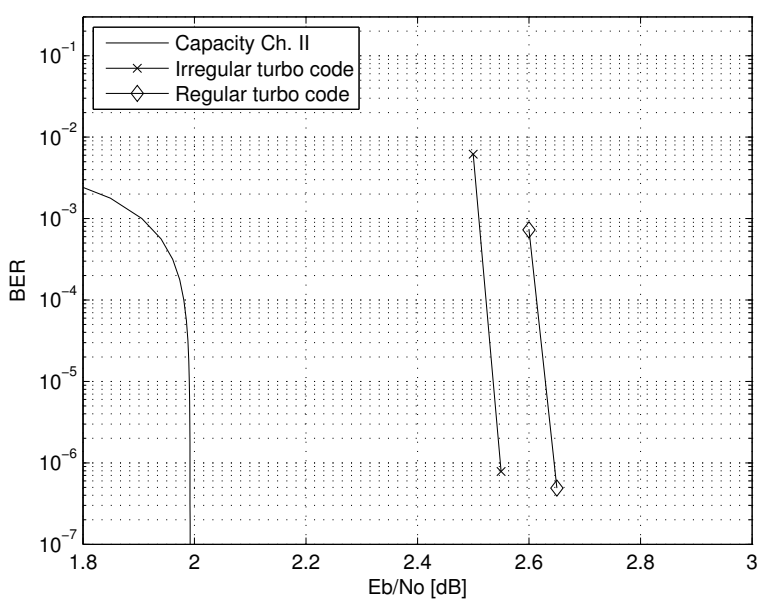

Fig. 6. Turbo equalization BER results for Channel II.

$10^{-6}$, which is only $0.55 \mathrm{~dB}$ from the channel capacity. The turbo equalizer that employs the regular turbo code achieves a similar BER at $E_{b} / N_{0}=2.65$, which is again about $0.1 \mathrm{~dB}$ more than its irregular counterpart. The number of simulated blocks was 1015 for the irregular code, and 1288 for the regular code. In this scenario, we set the number of inner iterations to be $n_{\text {in }}=5$ for $n_{\text {out }}$ between 1 and 7 and $n_{\text {in }}=20$ for $n_{\text {out }}>7$. Because different generator polynomials produce different $T_{D}(x)$, a different number of inner iterations is required for different codes.

The simulation results are consistent with the EXIT chart observations described before. For both channels, a decrease of $E_{b} / N_{0}$ by about $0.1 \mathrm{~dB}$ closes the bottleneck for the irregular turbo code. This means that the turbo equalizer can approach the capacity at the expense of a significantly higher number of outer iterations because the trajectory steps become much smaller for very narrow bottleneck openings. 


\section{CONClusion}

In this paper, we have analyzed a turbo equalization system that combines MAP equalization and irregular turbo codes. Our goal was to approach the information capacity limit for channels with severe ISI. To this end, we have performed an optimization of the degree profile of the employed irregular turbo codes. Although we have limited our optimization to profiles with two different degrees only, the analysis is applicable to any number and any combination of degrees. The simulation results show that the optimized irregular turbo codes outperform regular turbo codes by about $0.1 \mathrm{~dB}$ for the considered scenarios.

\section{REFERENCES}

[1] J. Proakis, Digital Communications, 4th ed. New York: McGraw-Hill, 2000.

[2] S. Lin and D. Costello, Error Control Coding, 1st ed. Englwood Cliffs, New Jersey: Prentice Hall, 1983.

[3] C. Douillard, M. Jezequel, C. Berrou, A. Piccart, P. Didier, and A. Glavieux, "Iterative correction of intersymbol interference: Turbo equalization," Europ. Trans. Commun., vol. 6, no. 5, pp. 507-511, Sep. 1995.

[4] C. Berrou, A. Glavieux, and P. Thitimajshima, "Near shannon limit errorcorrecting coding: Turbo codes," in Proc. IEEE International Conference on Communications, ICC '93, Geneva, Switzerland, May 1993, pp. 1064-1070.

[5] G. Bauch, H. Khorram, and J. Hagenauer, "Iterative equalization and decoding in mobile communications systems," in Proc. European Personal Mobile Communication Conference, EPMCC '97, Munich, Germany, Sep. 1997, pp. 307-312.

[6] S. Benedetto, D. Divsalar, G. Montorsi, and F. Pollara, "Serial concatenation of interleaved codes: Performance analysis, design, and iterative decoding," IEEE Trans. Inf. Theory, vol. 44, no. 3, pp. 909-926, May 1998.

[7] A. Glavieux, C. Laot, and J. Labat, "Turbo equalization over a frequency selective channel," in Proc. International Symposium on Turbo Codes, Brest, France, Sep. 1997, pp. 96-102.
[8] C. Laot, A. Glavieux, and J. Labat, "Turbo equalization: Adaptive equalization and channel decoding jointly optimized," IEEE J. Sel. Areas Commun., vol. 9, no. 9, pp. 1744-1752, Sep. 2001.

[9] M. Tuchler, R. Koetter, and A. Singer, "Turbo equalization: Principles and new results," IEEE Trans. Commun., vol. 50, no. 5, pp. 754-767, May 2002.

[10] M. Tuchler, A. Singer, and R. Koetter, "Minimum mean squared error equalization using a priori information," IEEE Trans. Signal Process., vol. 50, no. 3, pp. 673-683, Mar. 2002.

[11] V. D. Trajkovic, P. B. Rapajic, and R. A. Kennedy, "Turbo DFE algorithm with imperfect decision feedback," IEEE Signal Process. Lett. vol. 12 , no. 12 , pp. $820-823$, Dec. 2005.

[12] D. Raphaeli and Y. Zarai, "Combined turbo equalization and turbo decoding," in Proc. IEEE Global Communication Conference, GLOBECOM '97, Phoenix, USA, Nov. 1997, pp. 639-643.

[13] W. Ryan, L. McPheters, and S. McLaughlin, "Combined turbo coding and turbo equalization for PR4-equalized lorentzian channels," in Proc. 1998 Conf. on Inf. Sciences and Systems, Princeton University, USA, 1998.

[14] M. Tuchler, "Convergence prediction for iterative decoding of threefold concatenated systems,' in Proc. Globecom '02, Taipei, China, Nov. 2002, pp. $1358-1362$.

[15] S. ten Brink, "Convergence behavior of iteratively decoded parallel concatenated codes," IEEE Trans. Commun., vol. 49, no. 10, pp. 1727 1737, Oct. 2001

[16] B. J. Frey, Graphical Models for Machine Learning and Digital Communication, 1st ed. Cambridge, Massachusetts: The MIT Press, 1998.

[17] D. Arnold and H. A. Loeliger, "On the information rate of binaryinput symetric channels," in Proc. IEEE International Conference on Communications, Zurich, Switzerland, 2001, pp. 2692-2695.

[18] J. Hagenauer, E. Offer, and L. Papke, "Iterative decoding of binary block and convolutional codes," IEEE Trans. Inf. Theory, vol. 42, no. 2, pp. 429-445, Mar. 1996.

[19] M. Tuchler and J. Hagenauer, "Exit charts of irregular codes," in presented at Proc. CISS. [CD-ROM], Berlin, Germany, Jan. 2002, pp. 53-60.

[20] M. Fu, "Stochastic analysis of turbo decoding," IEEE Trans. Inf. Theory, no. 1, pp. 81-100, Jan. 2005.

[21] J. Boutros and G. Caire, "Iterative multiuser joint decoding: Unified framework and asymptotic analysis," IEEE Trans. Inf. Theory, vol. 48, no. 7, pp. 1772-1793, Jul. 2002 